CAHIERS DE

NARRATOLOGIE

\section{Cahiers de Narratologie}

Analyse et théorie narratives

25 | 2013

Humour et modernité dans les littératures de langues romanes du XIXe au XXIe siècle

\title{
Narrateur, personnage et lecteur. Pragmatique des subjectivèmes relationnels, des points de vue énonciatifs et de leur dialogisme
}

\section{Emilie Goin}

\section{OpenEdition}

\section{Journals}

Édition électronique

URL : http://journals.openedition.org/narratologie/6797

DOI : 10.4000/narratologie.6797

ISSN : 1765-307X

Éditeur

LIRCES

Référence électronique

Emilie Goin, « Narrateur, personnage et lecteur. Pragmatique des subjectivèmes relationnels, des points de vue énonciatifs et de leur dialogisme », Cahiers de Narratologie [En ligne], 25 | 2013, mis en ligne le 20 décembre 2013, consulté le 23 avril 2019. URL : http://journals.openedition.org/ narratologie/6797; DOI : 10.4000/narratologie.6797

Ce document a été généré automatiquement le 23 avril 2019

Article L.111-1 du Code de la propriété intellectuelle. 


\title{
Narrateur, personnage et lecteur. Pragmatique des subjectivèmes relationnels, des points de vue énonciatifs et de leur dialogisme
}

\author{
Emilie Goin
}

1 Nous nous proposons d'ouvrir quelques pistes dans l'étude des relations énonciatives et pragmatiques (principalement idéologiques et affectives) entre les instances internes du récit que nous choisissons de nommer narrateur ${ }^{1}$, personnage ${ }^{2}$ et lecteur virtuel ${ }^{3}$. Ces relations peuvent être rassemblées sous la forme d'un système pour lequel nous allons proposer une modélisation sous le nom de système intra-relationnel du récit. À la lumière de l'analyse du roman La Femme de Gilles de Madeleine Bourdouxhe, où les points de vue $\mathrm{du}$ narrateur et du personnage s'articulent de manière particulièrement intéressante ${ }^{4}$, nous étudierons de plus près les subjectivèmes relationnels en nous penchant essentiellement sur les problèmes suivants : leur repérage, l'identification de leurs effets pragmatiques, leur assemblage en un PDV énonciatif émanant d'un sujet énonciateur particulier, les différentes articulations possibles entre les PDV du narrateur et du personnage et leurs effets pragmatiques.

[Image non convertie]Le système des relations pragma-énonciatives entre le narrateur, le personnage et le lecteur virtuel peut être modélisé comme suit : 


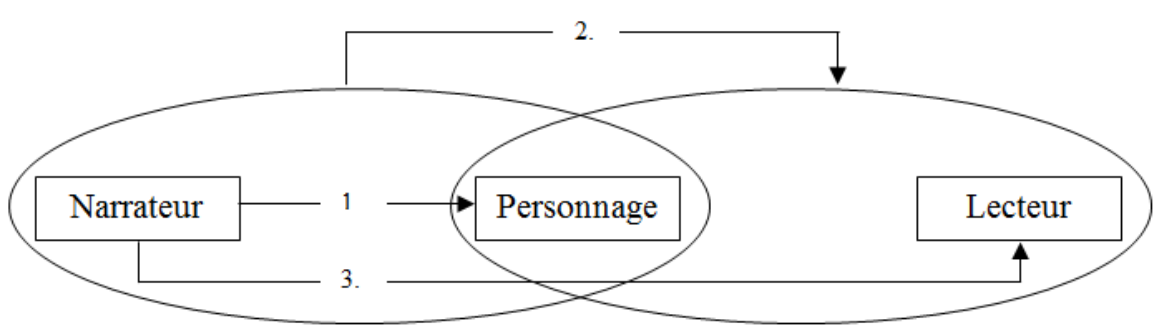

3 Rappelons qu'avec ce modèle, nous entendons rendre compte des récits à narrateur extra-diégétique, où l'effet-narrateur et l'effet-personnage sont particulièrement prégnants, autorisant ainsi la reconstruction et la confrontation des idéologies et affects de ces instances. Il existe bien entendu de nombreuses transgressions des codes relationnels généraux que nous tentons de dégager ici.

4 (1) La relation narrateur-personnage : le narrateur se positionne de manière critique et affective par rapport à ce qu'il advient du personnage. De manière générale, tous les jugements et marques d'affect que le narrateur émet dans le récit (de manière explicite ou implicite) ont un impact sur cette relation. Les jugements permettent de reconstruire l'idéologie du narrateur (entendue comme système de valeurs), idéologie dans laquelle il faut situer les valeurs attribuées au personnage principal. En ce qui concerne les marques d'affect, nous identifierons quelques procédés suscitant l'empathie par l'identification. Nous verrons également comment les phénomènes de prise en charge du PDV du personnage peuvent être source de jugements ou d'empathie.

(2) L'impact de la relation narrateur-personnage sur la relation lecteur-personnage : les jugements que le narrateur pose directement ou indirectement sur le personnage ont un impact sur le lecteur, impact que Vincent Jouve nomme effet idéologique (1992: 102) : le lecteur, confronté à l'idéologie que le narrateur désire lui faire admettre, interroge son propre système de valeurs. Qu'il adhère ou non au jugement du narrateur, celui-ci influencera nécessairement son propre jugement sur le personnage. De la même manière, les marques d'affect, explicites ou implicites, peuvent influencer le lecteur en sollicitant son pathos, c'est ce que nous appelons l'effet pathémique. Cet effet est généré, au-delà des marques d'affect, par le processus d'identification, qui peut prendre diverses formes selon les procédés énonciatifs et narratifs utilisés. Des effets escomptés sur le lecteur dépendent aussi les choix des mécanismes de prise en charge des paroles, pensées et perceptions du personnage par le narrateur.

6 (3) La relation narrateur-lecteur: le narrateur étant doté d'un PDV propre, il peut émettre des commentaires générateurs d'effets idéologiques et pathémiques sans que les jugements et marques d'affect ne portent sur le personnage ou ne soient exprimés au travers du PDV de ce dernier. Cette relation peut cependant avoir un impact sur la représentation de la relation (1) et sur les effets pragmatiques de celle-ci.

7 Nous n'avons pas représenté de vecteur lecteur $\rightarrow$ narrateur. Il est entendu que le positionnement idéologique et affectif du lecteur virtuel vis-à-vis du narrateur, du personnage, de leur relation et de la diégèse en général est pris en compte par le narrateur en termes d'effets pragmatiques attendus qui déterminent toute la construction du récit et des relations susdites. 
Ces trois vecteurs, qui ensemble composent le système intra-relationnel, ne seront pas étudiés chacun de manière isolée; nous verrons plutôt comment un même mécanisme discursif peut se situer au croisement de plusieurs vecteurs, voire de tous.

9 En appliquant l'analyse intra-relationnelle au récit La Femme de Gilles, nous rencontrons plusieurs difficultés dont le dépassement nécessite la mise au point de divers outils. Pour déterminer comment le narrateur se positionne idéologiquement et affectivement par rapport au personnage (relation 1), il nous faut tout d'abord repérer les unités du discours qui comportent les marques subjectives susceptibles de nous intéresser dans l'étude de cette relation, ce que nous nommons les subjectivèmes relationnels. Ces subjectivèmes doivent par ailleurs être assemblés de manière à constituer un PDV identifiable du narrateur sur le personnage. Ce PDV doit enfin être considéré dans sa dimension pragmatique, de manière à déterminer comment la relation narrateurpersonnage produit des effets principalement idéologiques et pathémiques susceptibles d'influencer la relation lecteur-personnage (et au-delà les relations lecteur-narrateur et lecteur-récit dans son ensemble).

\section{Le repérage des subjectivèmes}

10 Tout dans le langage est, d'une certaine manière, subjectif puisque pour désigner un objet du monde le sujet parlant choisit des mots et tournures de phrases dans une série de paradigmes prévus par le code, ce choix pouvant varier suivant la personnalité du sujet mais aussi suivant le contexte d'énonciation. De là, n'importe quelle unité langagière est susceptible de devenir subjective lorsqu'elle est employée en discours. Au-delà de ce simple postulat, il s'avère intéressant de déterminer quels sont les éléments du discours qui sont plus subjectifs que d'autres et surtout lesquels sont susceptibles de nous donner des informations sur la relation que le locuteur entretient avec l'objet qu'il désigne.

11 Cet aspect graduel de la subjectivité est souligné par Kerbrat-Orecchioni dans son étude de l'énonciation (1980). Or pour elle, la subjectivité d'un adjectif serait d'autant plus accentuée que les contours de sa classe dénotative sont flous. Ainsi, il serait plus facile d'obtenir un consensus sur l'appartenance d'un $\mathrm{x}$ à la classe des célibataires ou des objets jaunes, que sur son appartenance à la classe des hommes bons (1980: 80). Toutefois, si nous imaginons quel pourrait être l'emploi de ces termes en contexte, célibataire est, selon nous, plus fréquemment subjectif que jaune parce qu'il peut être utilisé pour véhiculer un sous-entendu. D'où l'intérêt de décrire non pas le degré de subjectivité contenu dans les éléments de la langue, mais le potentiel pragmatique des marques subjectives (ou subjectivèmes) employées en discours. Nous nous centrerons sur les subjectivèmes axiologiques et affectifs qui, de ce fait, sont porteurs d'effets idéologiques et affectifs, tant au niveau micro- que macro-textuel.

12 Kerbrat-Orecchioni définit les axiologiques et les termes affectifs comme étant porteurs d'une double information; en même temps que de qualifier le référent, ils informent sur la relation que le sujet énonciateur entretient avec l'objet dénoté. Ainsi, en exprimant la position favorable ou défavorable du locuteur vis-à-vis de l'objet qualifié, l'axiologique donne une indication sur le système de valeurs de ce locuteur et sur les normes personnelles qui sous-tendent ce système. Les traits affectifs quant à eux informent sur la relation émotionnelle que le locuteur entretient avec l'objet dénoté. Cependant lorsqu'il s'agit de trouver un moyen de repérer ces traits axiologiques et affectifs, Kerbrat- 
Orecchioni bute contre le caractère purement formel de son système d'analyse : d'une part un terme neutre peut devenir axiologique ou pathémique par l'usage de l'ironie ou par l'action du contexte verbal (1980:85-88), d'autre part, ces traits peuvent avoir d'autres supports que les unités lexicales, par exemple, les constructions syntaxiques, les effets de rythmes, la typographie, l'organisation des faits et des sèmes (parallélismes, oppositions, analogies), etc. Par ailleurs, les traits affectifs chevauchent les traits axiologiques, puisqu'un jugement proféré est souvent intimement lié à une émotion ressentie (1980 : 96). Prenons l'exemple suivant :

Elle, avec ce goût de cendre dans la gorge, regarde l'éternelle fenêtre glauque et la lueur jaunâtre que la veilleuse répand sur les choses. (p. 48)

Les unités lexicales de cet extrait ne comportent pas de sème strictement axiologique ou affectif. Mais « glauque » et « jaunâtre » désignent respectivement un vert foncé, souvent associé aux eaux troubles, et un jaune pâle, souvent utilisé dans un sens péjoratif, par exemple pour un objet jauni par le temps. Ces termes sont donc porteurs de traits axiologiques et pathémiques qui leur sont attachés grâce à un savoir de type encyclopédique (cf. Eco 1985). Ces connotations péjoratives sont d'autant plus pathémiques que les adjectifs apparaissent dans une perception d'un personnage dont on suggère au préalable le mal-être. En effet, si le nom « cendre » n'est pas non plus porteur, en langue, d'un jugement ou d'un affect particulier, lorsqu'il devient le complément du nom "goût", il ne peut véhiculer qu'une représentation imagée, symbolisant doxiquement un mal-être éprouvé. C'est donc le cotexte et le contexte qui confèrent au nom « cendre » ses connotations axiologique et pathémique. Les subjectivèmes dégagés ne sont donc pas codés en langue, c'est le cotexte et le contexte qui sont pourvoyeurs de cette subjectivité identifiable à travers les effets pragmatiques visés par le discours.

Au niveau macro-textuel, la subjectivité relationnelle peut s'exprimer, par exemple, à travers l'organisation des faits du récit. Il s'agit dès lors d'une subjectivité implicite. Or, si l'on observe que le rapprochement de certains faits est subjectif, c'est avant tout parce que les parallélismes et/ou oppositions ainsi créés sont susceptibles de produire des effets idéologiques et/ou pathémiques. Prenons l'exemple suivant :

(1) (...) Elle portait fièrement, bien en avant, ce poids nouveau qui lui venait du corps de Gilles.

Il rentra, un peu en retard, accompagné de Victorine. (p. 21)

La juxtaposition des faits contenus dans ces deux phrases génère une opposition entre un acte valorisant d'Élisa vers Gilles, porter son fils la rend fière, et un acte outrageant de Gilles vers Élisa, revenir à la maison avec son amante. De plus, la paralipse qui cache l'information « tromperie » et ne fait que la suggérer au moyen du « retard », a un double effet : elle génère à la fois une complicité entre les instances détentrices du savoir (le narrateur, le récepteur et les amants) et de l'empathie pour le personnage exclu du savoir, à qui cette tromperie fait l'affront. Cette opposition génère un effet idéologique et pathémique : la tromperie de Gilles doit paraitre d'autant plus ignoble au récepteur que l'amour d'Élisa pour son mari est grand. Cette sensation de comble est encore appuyée par la reprise anaphorique «il», malgré l'ouverture d'un nouveau paragraphe. Cette reprise mime formellement l'idée que c'est ce même «corps» de Gilles, adulé par Élisa, qui commet cet affront.

Le repérage des subjectivèmes relationnels implicites est donc lié à l'identification de leurs effets pragmatiques. 


\section{Pragmatique des subjectivèmes}

17 Il s'agira ici d'identifier quelques constantes dans la production des effets pathémiques et idéologiques ${ }^{5}$.

\section{L'effet pathémique}

18 On a vu que l'émotion pouvait être exprimée de manière explicite (au moyen de sèmes traduisant un affect), ou de manière implicite (en convoquant des représentations ou des situations doxiquement rattachées à des émotions). De manière plus générale, d'autres mécanismes peuvent être mis en place pour que le récepteur s'identifie au personnage ou pour que du moins il éprouve de l'empathie pour ce dernier.

La notion d'identification fait débat dans les théories de la réception du récit. La définition structurale qu'en donne Barthes est généralement sélectionnée comme base de réflexion : «L'identification (...) est une pure opération structurale : je suis celui qui a la même place que moi » $(1977: 153)$. Mais cette affirmation est extrêmement vague et donne lieu à de multiples interprétations et réaménagements. On peut identifier quatre types d'identification résultant de ces différentes interprétations: l'identification focalisatrice, l'identification informationnelle, l'identification émotionnelle et l'identification heuristique.

Jouve reprend à son compte la définition de Barthes avant de l'adapter comme suit: "Celui qui est à la même place que moi, c'est (...) celui qui voit de la même place que moi. » (1992 : 124). Le lecteur s'identifierait donc à l'instance qui focalise (au sens de Bal) le récit, autrement dit, à l'instance à l'origine du PDV. Nous appellerons ce phénomène, l'identification focalisatrice. Jouve nous dit en effet « qu'en imposant au lecteur son point de vue sur l'histoire, le narrateur l'oblige du même coup à entrer dans son jeu. » (1992:125) Il poursuit en donnant des exemples de récit où le lecteur est amené à s'identifier à un narrateur tantôt chrétien, tantôt athée. L'identification n'est donc pas pour lui purement visuelle (comme le laisse entendre sa première définition); elle consiste également à adopter le prisme idéologique et émotionnel de l'instance qui perçoit. C'est dans la même optique que Rabatel reprend la citation de Barthes en précisant que le lecteur ne s'identifie pas seulement à celui qui agit mais aussi à celui qui perçoit, parle ou raconte «à la même place que moi» (2010a:132). Notons qu'encore une fois le sens de cette formulation est flou. Nous le comprenons comme suit : « Je suis susceptible de me mettre à la place de celui qui agit, perçoit, parle ou raconte », qu'il s'agisse du narrateur ou du personnage.

21 Pour Jean-Louis Dumortier, l'identification narratoriale est impossible, car le lecteur s'identifie non pas à un PDV mais «à celui qui, dans la fiction narrative ou dans la diégèse, se trouve, s'agissant du savoir, dans une situation analogue à la [s]ienne » (2001 : 152). Le lecteur ne peut donc s'identifier au narrateur puisque celui-ci en sait toujours plus que celui-là sur l'histoire qu'il est en train de raconter. L'identification reposant ici sur les mécanismes du savoir, nous la qualifierons, à la suite de Jouve, d'informationnelle (1992 : 129). D’après notre conception du narrateur extra-diégétique, il est évident que celui-ci est toujours censé avoir un rapport d'omniscience à l'histoire qu'il raconte, du moins dans le cadre des récits fictifs. Le fait est que l'identification informationnelle ne s'effectue pas par rapport à la détention théorique du savoir, mais par rapport aux 
informations effectivement transmises ou non transmises par le narrateur et les personnages. Les dissimilitudes de savoir entre les instances sont alors à observer comme des mécanismes stratégiques visant à obtenir certains effets pragmatiques sur le lecteur. On observe deux cas de figure selon qu'on se situe dans le PDV du narrateur ou du personnage : soit le narrateur retient son savoir pour faire mine de partager (et faire partager) les doutes et questionnements du personnage, ainsi que les émotions liées à ce non-savoir (identification informationnelle et émotionnelle), soit le narrateur représente le non-savoir d'un personnage tout en montrant qu'il en sait plus. Dans ce deuxième cas, le narrateur et le lecteur peuvent éventuellement partager ce savoir avec un ou plusieurs autres personnages, à l'exclusion d'un ou plusieurs autres personnages. Dans tous les cas, un rapport de complicité s'établit entre les instances qui partagent le même savoir, complicité plus ou moins bien tolérée par le lecteur suivant que le texte l'a habitué à juger positivement ou négativement, à ressentir de l'empathie ou non, vis-à-vis des personnages excluant et exclus. Voici deux exemples qui, à ce niveau, produisent des effets inverses :

(2) [Élisa se doute de l'adultère de Gilles. Une nuit, Gilles s'absente de la maison, sous prétexte d'aller se balader. Élisa décide de le suivre, mais il prend un chemin inhabituel. Élisa perd sa trace et rentre à la maison. Au retour de Gilles, elle lui pose des questions d'apparence innocente: ]

- Tu as bien pris l'air ! dit Élisa. Où es-tu allé ?

- D'abord chez Gobelet, quelques minutes... puis je suis descendu en ville, question de voir un peu d'animation...

- Ah !...

Et elle le revit aussitôt sortant de la boulangerie et marchant sur la route en tournant le dos à la ville...

- Tes souliers sont trempés... déchausse-toi... Elle prit derrière le fourneau des pantoufles chaudes qu'elle lui tendit:

- C'est en ville que tu t'es mouillé ainsi ?

- Non... mais rien que de remonter la route, ça suffit.

- Évidemment... Gilles s'étire, étend les jambes, remue un peu les pieds dans les pantoufles chaudes:

- Il fait bon ici... dit-il, j'ai faim...

- C'est prêt... (pp. 44-45)

(3) [À ce moment Élisa ne sait pas encore que Gilles la trompe, mais le lecteur a déjà assisté au début de la relation adultère.]

(...) Elle portait fièrement, bien en avant, ce poids nouveau qui lui venait du corps de Gilles.

Il rentra, un peu en retard, accompagné de Victorine.

- J'ai ramené la petite...dit-il. Elle avait l'air de s'ennuyer à la maison, et comme tu ne sors plus guère, j'ai pensé que je pourrais peut-être aller faire un tour avec elle ce soir...

- Tu as bien fait, répondit Élisa.

Elle regarda la jeune fille. Elle fut fière de la voir si jolie et si fraîche, et songeant à son corps à elle, chaque jour plus lourd et plus déformé, elle se dit : « C'est très bien qu'il sorte avec elle, ça le distraira. » (p. 21)

En (2), le lecteur, le narrateur et Élisa partagent une information que le second personnage ne détient pas: Gilles ne sait pas qu'Élisa sait qu'il ment. Ce savoir crée un lien de complicité entre les instances qui se trouvent en quelque sorte liguées contre Gilles. Le partage du savoir «Gilles ment " permet l'élaboration de sous-entendus. Par exemple, l'ironie contenue dans le «Évidemment... », qui arrive juste après l'évidence du mensonge de Gilles, unit le narrateur, le personnage et le lecteur dans un jugement et une forme de moquerie à l'encontre de Gilles. 

affective et idéologique que le lecteur virtuel est censé nouer avec ces deux premières instances. Dans bien des cas, les effets pathémiques servent à appuyer l'idéologie véhiculée par le récit.

\section{L'effet idéologique}

27 Lorsqu'il est confronté à l'idéologie (entendue comme système de valeurs ou système axiologique) de l'émetteur, le récepteur est amené à remettre en question ses propres schèmes conceptuels; c'est ce que nous appelons, à la suite de Jouve (1992: 102-105), l'effet idéologique.

Cette idéologie est particulièrement identifiable lorsque le narrateur attribue des valeurs aux personnages. Selon Philippe Hamon, un personnage peut être jugé dans sa faculté à 
manipuler des "canons esthétiques ", des "signes linguistiques ", des « outils » et des «lois», soit le "savoir-jouir» (perceptions), le «savoir-dire» (échanges verbaux), le «savoir-faire» (travail) et le "savoir-vivre» (conduite sociale) (1984: 24, 1136). Le narrateur peut marquer positivement ou négativement l'un ou plusieurs de ces savoirs, soit en mentionnant explicitement son jugement, soit implicitement, par exemple en opposant l'un à l'autre de manière récurrente un savoir marqué axiologiquement et un savoir non marqué. Selon Jouve (1992: 102-105), c'est de ce marquage que dépend l'effet idéologique de l'œuvre. À cela, nous aimerions ajouter que les personnages aussi peuvent construire un système axiologique en portant des jugements sur les autres personnages. Pour déterminer le sens à donner au récit, il faut donc analyser les relations d'accord et de confrontation entre l'idéologie du narrateur et celle du personnage. Mais de nouveau, le problème de l'hétérogénéité énonciative se pose. Comment savoir si les jugements repérés appartiennent au personnage ou au narrateur? Et lorsqu'ils appartiennent au personnage, comment savoir si le narrateur, qui est tout de même le rapporteur de ces jugements, $y$ adhère ou non. Pour pouvoir illustrer ces remarques concernant l'effet idéologique, il nous faut donc décrire les outils qui nous serviront à l'analyse et à l'interprétation de l'hétérogénéité énonciative.

Lorsqu'il s'agit de rattacher les subjectivèmes à une subjectivité particulière, l'analyse du discours emploie généralement le modèle de la perception visuelle avec des concepts tels que la focalisation ou le PDV.

\section{L'assemblage des subjectivèmes en PDV}

Selon Rabatel (2004), en effet, une analyse des points de vue ne peut se contenter de rechercher dans les textes un "foyer " à partir duquel les objets seraient perçus et référenciés (comme le fait Genette), parce que le PDV n'est jamais indissociable de l'objet perçu. Notre perception du monde ne peut être objective étant donné qu'elle s'appuie toujours sur des constructions intellectualisées en perpétuelle évolution. Ces constructions que B. Pottier nomme schèmes conceptuels sont des mécanismes cognitifs sous-jacents aux catégorisations opérées par les langues pour désigner les objets du monde $\left(2001: 4^{7}\right)$. Il y a donc a priori une subjectivité cognitive qui précède la subjectivité langagière de la référenciation. Ainsi, puisque « en changeant ce qu'il connaît du monde, l'homme change le monde qu'il connaît » (Dobzhansky 1966 : 391), même si le but du sujet parlant est de référer aux objets du monde (donné phénoménal ou noématique), il ne peut en réalité référer qu'à la perception subjective qu'il a de ce monde (noétique) (cf. P. Ouellet et alii 1994: 135-156). Nous pouvons donc schématiser le processus de référenciation comme suit : 


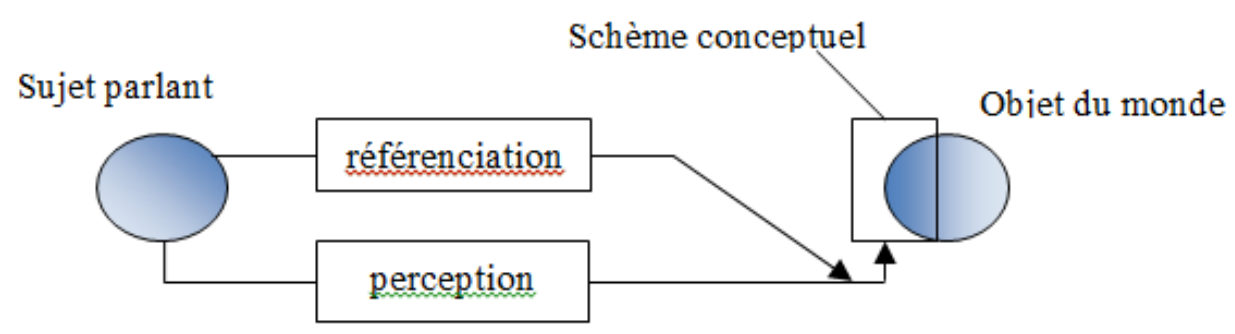

31 Si l'on applique ce schéma au récit fictionnel, en considérant que le sujet parlant est le narrateur et que l'objet du monde est le référent virtuel auquel renvoient les mots de ce narrateur, le processus de référenciation représente le récit. Dès lors, lorsqu'il construit linguistiquement les objets du monde fictif, le narrateur (fictif lui aussi) se construit luimême en tant que sujet percevant : il donne au lecteur des indications sur la façon dont il perçoit le monde à travers ses propres schèmes conceptuels.

C'est à partir de cette « référenciation des objets du discours coréférant à l'énonciateur » (Rabatel 2000 : 52) que Rabatel construit sa notion de PDV qu'il définit comme suit :

On nommera PDV tout ce qui, dans la référenciation des objets (du discours) révèle, d'un point de vue cognitif, une source énonciative particulière (locuteur/ énonciateur ou énonciateur) et dénote, directement ou indirectement, ses jugements sur les référents - d'où l'importance des dimensions axiologiques et affectives du PDV. (2009: 7 )

Le PDV se révèle être un outil plus adéquat que les focalisations genettiennes ${ }^{8}$ par exemple pour étudier la représentation que le narrateur donne de sa relation au personnage et l'impact pragmatique de cette représentation sur la relation que le lecteur virtuel est censé nouer avec le narrateur et le personnage. Prenons un exemple :

(4) Quelques jours après, Élisa revit Victorine. C'était son jour de congé, on l'avait remplacée au magasin, disait-elle, et c'est ainsi qu'elle avait pu monter jusqu'ici un matin pour dire bonjour.

Elle parlait, accoudée au buffet de la cuisine. Avec son visage coloré d'un fard à bon marché, son faux renard noir, son grand chapeau de paille ça peut se mettre hiver comme été - elle perdait toute fraicheur et toute beauté.

Élisa la regardait tristement, elle aurait voulu lui dire qu'avec cet air de fausse élégance elle faisait peine à voir. Mais elle paraissait si fière de ces loques!

Élisa se mit à laver la vaisselle, en écoutant le bavardage de Victorine, la tête penchée sur sa besogne. (p. 499)

Le système des focalisations genettiennes nous permet d'identifier le passage souligné comme une focalisation interne sur le personnage d'élisa, le reste de l'extrait relevant plutôt d'une focalisation externe. Une théorie basée sur le concept du PDV nous permet en revanche d'expliquer plusieurs phénomènes d'imbrications des perspectives. Tout d'abord, le paragraphe en italique peut être considéré comme un compte rendu des perceptions d'Élisa (ce que Rabatel nomme PDV représenté, cf. infra). Cependant, comme le verbe de perception « regard[er] » et les jugements attribués explicitement à élisa sont placés postérieurement, le narrateur ménage une ambiguïté quant à la source énonciative des jugements contenus dans le passage en italique. Cette ambiguïté laisse entendre que 
les valeurs qui fondent ces jugements (contre la fausse élégance) sont communes au narrateur et au personnage. Qui plus est, ces valeurs sont alors celles que le narrateur entend communiquer au lecteur (effet idéologique). Deux autres phénomènes (que les focalisations genettiennes ne peuvent mettre en évidence) viennent confirmer cette interprétation. La proposition incise "ça peut se mettre hiver comme été » exprime, en vertu de son caractère général, un PDV doxique (opinion commune) auquel adhère Victorine, puisqu'il justifie une pratique de ce personnage (porter un chapeau de paille en hiver). Cette proposition fait éventuellement référence à une parole qu'aurait pu tenir la jeune fille. Dans tous les cas, ce PDV est rapporté ironiquement par le narrateur (et Élisa, si on considère qu'on est dans un PDV représenté), puisque les autres jugements du cotexte sont radicalement négatifs. L'usage de l'ironie (le fait de rapporter le PDV du personnage pour le retourner contre lui) permet d'établir une complicité avec le destinataire par la moquerie (effet à la fois idéologique et pathémique). Enfin, la dernière phrase ne relève pas d'un simple PDV externe et objectif. La juxtaposition des faits " Victorine qui bavarde accoudée au buffet parée de tous ses accessoires de beauté » et "Élisa qui écoute, la tête penchée sur sa vaisselle », a pour but de relever une opposition axiologiquement marquée entre les comportements sociaux des personnages: la dévalorisation de la jeune fille insouciante induit une valorisation implicite de la mère au foyer qui travaille. En opposant ces comportements, le narrateur entend également mettre en évidence le culot de Victorine qui, bien qu'elle entretient une relation avec le mari d'Élisa, parle néanmoins à cette dernière comme si de rien n'était. Il y a donc un jugement implicite et un effet idéologique forts qui se dégagent de cette situation.

D'après cette analyse, les idéologies du personnage et du narrateur seraient donc en accord pour juger négativement la conduite sociale de Victorine. Mais pour pouvoir rendre compte plus clairement de ces relations entre PDV il nous faut développer plus en avant la problématique de l'hétérogénéité énonciative.

\section{L'hétérogénéité énonciative}

Comme nous l'avons vu dans l'analyse de l'extrait précédent, le concept de PDV s'avère être un outil adéquat pour analyser les phénomènes d'hétérogénéité énonciative, c'est-àdire le fait qu'un énoncé puisse contenir les PDV de plusieurs énonciateurs pris en charge par un même locuteur. Pour Ducrot, le terme énonciateur ne désigne que l'instance garante d'un PDV à l'intérieur de l'énonciation du locuteur (1984 : 204-205). Chez Rabatel, par contre, le locuteur est, lui aussi, un énonciateur puisqu'en prenant la parole il expose bien un PDV. Rabatel opère alors une nouvelle distinction entre locuteur / énonciateur premier (désormais L/E1) et l'énonciateur second (e2). Il pense en effet, à l'encontre de Ducrot, que c'est le L/E1 qui prend en charge les PDV auxquels il adhère et qui impute aux e2 les PDV par rapport auxquels il ne fait que se positionner en marquant son accord, son désaccord ou sa neutralité (2008: 60-67).

Dans bien des cas, il est difficile de déterminer qui, du L/E1 ou de l'e2, est la source énonciative du PDV. Comme dans l'exemple précédent, on a tendance à interpréter ces cas d'ambigüité comme un accord à la fois idéologique et affectif entre les instances : non seulement leurs jugements convergent, mais en plus, ces instances tendent à être confondues en une seule, par une forme d'identification, ce qui suppose aussi un partage des affects. L'absence de mise à distance du PDV par L/E1, incite généralement le récepteur à adhérer, lui aussi, à l'idéologie et aux affects véhiculés par ce PDV. 
38 Les effets pragmatiques générés par le récit sont donc susceptibles de diverger suivant la manière dont les PDV du narrateur et du personnage sont articulés. C'est pourquoi il semble intéressant d'envisager un classement pragma-énonciatif des différents mécanismes d'articulation des PDV.

On peut catégoriser ces mécanismes en se référant aux différentes formes de discours rapporté qui peuvent être appliquées aussi bien aux comptes rendus de paroles qu'aux comptes rendus de pensées (cf. Genette 2007: 172-189). Le récit offre toutefois la possibilité de représenter un niveau pré-verbal de la vie intérieur des personnages. Plusieurs outils spécifiques ont d'ores et déjà été élaborés pour rendre compte de ces phénomènes : le PDV représenté de Rabatel (2008) pour analyser les comptes rendus de perceptions, le psycho-récit de Dorrit Cohn (1981) pour les descriptions développées de l'état psychique du personnage ${ }^{10}$ où domine le PDV du narrateur (du moins dans la formulation), et enfin le monologue intérieur (que Cohn nomme "monologue autonome »), pendant psychique du discours direct libre. Le discours indirect libre (DIL) peut également remplir cette fonction, mais il s'utilise aussi pour des paroles et pensées verbalisées.

40 Ces mécanismes offrent des possibilités variables d'articulation des PDV du narrateur et du personnage, possibilités que l'on peut schématiser en deux grandes propriétés: le débrayage énonciatif et le mimétisme (c'est-à-dire la capacité à donner une image mimétique des paroles ou des mouvements de pensée qui pourraient être ceux du personnage). Ces propriétés permettent de produire différents effets pragmatiques généraux sur lesquels repose le choix stratégique du mécanisme de prise en charge du PDV du personnage.

41 En restant dans la problématique des cas d'ambigüité concernant l'identification de la source énonciative du PDV, nous allons nous concentrer ici sur le cas du débrayage énonciatif.

Le débrayage énonciatif consiste à signaler la présence du PDV d'un e2, à l'intérieur de l'énoncé de L/E1, au moyen de marques externes et internes. Les marques internes correspondent à l'ensemble des subjectivèmes repérables (cf. Kerbrat 1980, Rabatel 2008 : 77-79) ; les marques externes sont quant à elles déterminées par le type de mécanisme de prise en charge.

43 Les discours rapportés qui présentent les marques externes les plus évidentes sont bien entendu le discours direct (DD) et, dans une moindre mesure, le discours indirect (DI). Dans le cas du DI, bien que le narrateur surajoute toujours son PDV ${ }^{11}$ au PDV du personnage dont il rapporte un équivalent sémantique sans reproduire ses modalités d'énonciation, les sources énonciatives ne sont pas confondues puisque la prédication identifie la pensée comme étant celle du personnage.

44 En revanche, dans les variantes libres, soit le discours direct libre (DDL) et le discours indirect libre (DIL), l'absence de débrayage énonciatif amène le narrateur à tenir une part de responsabilité dans les éventuels jugements et marques d'affects émis. Ces types de discours rapporté constituent donc un terrain propice à un syncrétisme entre narrateur, personnage et lecteur. En effet, s'il prend part à l'idéologie et aux affects du personnage, le narrateur entend produire sur le destinataire des effets idéologiques et pathémiques du même ordre. Prenons tout d'abord un exemple de DDL.

(5) Elle [Élisa] le [Gilles] revit nettement, assis devant le feu, les jambes étendues, les pieds appuyés sur la porte du four ouvert, avec cet air de nouveau repu 
sommeillant. [...] « Ah oui, je dirai : si tu allais ramasser les tas avec la pelle ? et il dira : Ouf ! les tas, qu'est-ce que ça peut foutre... Et puis il aura cet air. Il... »

Il s'assoira avec cet air de type qui se met à l'aise, il reniflera, crachera sans retenue dans son mouchoir, et il sourira goulûment en fixant un point du fourneau. Ah oui, les tas, qu'est-ce que ça peut foutre.

«Mais non, c'est moi... tout me paraît drôle... c'est mon état. Est-ce que j'étais comme ça pour les jumelles? Pan! encore un petit coup de pied... en plein dans le ventre de sa mère... Eh bien ! ce sera un vigoureux celui-là... Oui... c'est plutôt moi... Eh bien ! allez... » (pp. 19-20)

Dans ce compte rendu des pensées d'Élisa, les DD (semblables à des monologues intérieurs du fait de leur déconstruction syntaxique, des pauses et des interjections) encadrent un passage qui peut être interpréter comme un DDL puisqu'il complète les pensées d'Élisa exprimées dans les DD et qu'il n'y a plus de débrayage énonciatif. Il peut également s'agir d'un commentaire du narrateur qui complète la pensée du personnage en la radicalisant. Le changement de régime s'accompagne en effet d'un changement de ton et de personnalité. Le deuxième DD qui commence par « Mais non, c'est moi... tout me paraitt drôle... c'est mon état. » indique toutefois qu'il y a bien une transformation psychique du personnage qui, lui-même, ne se sent pas comme à son habitude. Ce changement amène à la production d'axiologiques très négatifs, associés à une émotion de mépris et de dégoût, état d'esprit qui ne correspond pas du tout au caractère d'Élisa illustré dans cet extrait et dans l'ensemble du roman. Aussi, on peut se demander dans quelle mesure le narrateur ne superpose pas ses propres émotions (dégoût, mépris) à ce qui, chez la femme de Gilles, ne serait au départ qu'une simple rancœur. Quoi qu'il en soit, le fait que cette radicalisation des jugements et des émotions accompagnent le changement vers un cas d'ambigüité énonciative qui responsabilise le narrateur, montre que ce dernier cautionne ces jugements et émotions. Ce passage apparait, par opposition aux DD beaucoup plus évasifs, comme un moment de lucidité qu'Élisa aurait, pour une fois, atteint. Cette forme d'objectivation du jugement par l'adhésion du narrateur, ainsi que la netteté de la formulation qui apparaît comme une incise dominée par le narrateur au milieu des pensées éparses d'élisa, montrent que l'effet idéologique et pathémique escompté de ce passage est d'amener le destinataire à se représenter Gilles comme un goujat détestable et Élisa comme une femme (malgré tout) aimante qui essaye de fuir la réalité.

Dans le DIL, on peut parfois observer la dominance du PDV du narrateur ou du personnage, mais il est rarement possible d'attribuer à l'un ou l'autre un fragment de l'énoncé. D'après Rabatel (2008: 448), le DIL ne mélange pas seulement les voix mais aussi les espaces mentaux, les univers et les processus réflexifs des deux instances. L'indétermination porte aussi sur la nature des propos rapportés (paroles, pensées ou perceptions). Ces propos, décrits dans le premier plan, ne dépendent pas des capacités de verbalisation du personnage. Aussi, le narrateur peut très bien donner accès à un niveau de pensée infra-verbal, tout en juxtaposant ces pensées au récit d'évènements survenant eux aussi dans le premier plan, ce qui ajoute une indistinction entre extériorité et intériorité (cf. Cohn 1981 : 140-150). Observons les exemples suivants :

(6) Élisa redressa le buste et sourit, elle aussi, avec un amoureux orgueil. Il était à elle cet homme-là... Et elle l'aimait au point que, tout de même, elle avait bien le droit de le défendre, de le garder, à elle... à elle... Et rien n'y ferait... personne d'autre n'avait le droit... pas même lui de se détacher d'elle... Quoi qu'il arrivât, quoi qu'il fût arrivé, il ne fallait pas faire d'éclat. Seulement veiller, et n'agir qu'en de petits actes subtils, et garder intact cet amour autour de lui, et auquel il reviendrait : elle l'aimait, on n'échappe pas à un tel amour... (p. 41) 
(7) Que faisait-elle toute seule dans cette ombre froide? Un instant aveuglée par l'espoir, elle voulut se lever et retourner auprès des enfants. Elle s'en voulait maintenant de les avoir laissées seules.

Mais elle ne bougea pas. Elle regardait autour d'elle - et de toutes ces formes noyées de nuit, de ces lueurs voilées de brouillard il montait à nouveau une tristesse infinie.

Qu'importait que les enfants fussent seules! Elle les aimait de toute sa chair et de tout son cœur - elle ne pouvait aimer qu'ainsi - les deux petites filles blondes qui avaient les cheveux de Gilles et les yeux d'Élisa, et l'enfant qui était en elle et qu'elle sentait vivre contre son cœur. Mais cette femme qu'on dit mère entre les mères, ce n'est pas d'une chair et d'un cœur maternels qu'elle chérit son fruit. Enfants, continuation vivante d'un amour et qui ne possèdent tout leur prix que dans le rayonnement de cet amour... Enfants issus de l'époux et vivant dans la maison de l'époux. (pp. 39-40)

En (6), le DIL se rapproche formellement du DDL avec les mimétismes expressif (cf. les expressions relevant d'un sociolecte marqué : "Il était à elle cet homme-là...», la répétition du "tout de même») et psychique (les pauses, la répétition de certains syntagmes et du «et» qui miment une pensée ressassante). Le PDV du personnage domine au point que l'on se demande si le narrateur tient vraiment une responsabilité dans les contenus exprimés. Pourtant, grâce au PDV du narrateur, la formulation des pensées prend une tournure claire, surtout dans les deux dernières phrases. Si claire d'ailleurs, que ce DIL apparait comme un excellent justificatif d'un comportement d'Élisa difficile à comprendre : le choix de ne pas révéler qu'elle sait que son mari la trompe avec sa sœur, et de néanmoins tenter de le reconquérir. À travers ce DIL le narrateur exprime, non seulement un effort de compréhension des choix d'Élisa, mais également une volonté de les faire comprendre au lecteur, tout en restant proche du PDV du personnage au moyen des mimétismes. Le DIL semble être le mécanisme de prise en charge idéal pour construire ce type d'argumentation indirecte à deux voix, où le narrateur utilise le pathos du personnage (ainsi que des traits caractéristiques qui rappellent la condition sociale de ce dernier) pour convaincre le lecteur, non pas du bienfondé des actes du personnage, mais du moins de la légitimité de ses intentions et des valeurs (abnégation, courage) qui l'animent.

En (7), les DIL se bornent aux deux phrases courtes insérées dans des psycho-récits, où, par définition, le PDV du narrateur est plus présent. Ces deux phrases servent de tremplin aux émotions développées et expliquées dans les psycho-récits. L'interrogation et l'exclamation représentent en effet un sursaut émotionnel du personnage qui a également pour fonction d'interpeller le destinataire. De nouveau, on a une forme d'argumentation indirecte à deux voix, sauf que c'est le psycho-récit qui assure la fonction de justification tandis que le DIL exprime la position du personnage qu'il s'agit de défendre. Notons qu'il y a tout de même une indétermination quant à la source énonciative de ces deux phrases que nous interprétons comme étant des DIL. Il pourrait en effet très bien s'agir de commentaires du narrateur qui en (1) ferait mine de s'interroger sur les motivations du personnage et en (2) exprimerait avec force un avis destiné à défendre le comportement d'élisa. Dans les deux cas, l'effort de clarification et de justification de la part du narrateur montrent de nouveau que ce dernier tente de comprendre et de faire comprendre les choix du personnage, tout en utilisant le potentiel persuasif de l'empathie. Introduit par «Elle regardait autour d'elle », « et de toutes ces formes noyées de nuit, de 
ces lueurs voilées de brouillard il montait à nouveau une tristesse infinie » est un PDV représenté. Comme le souligne Rabatel (2008), ce type de prise en charge est souvent confondu avec le DIL parce qu'il est, lui aussi, caractérisé par la présence de formes de visée sécante et d'imparfaits à valeur subjective. Mais alors que le DIL peut exprimer une pensée autonome par rapport au monde extérieur dans lequel évolue le personnage, le PDV représenté est pour sa part intimement lié à une perception sensorielle de ce monde. C'est ce qui explique que, contrairement au DIL, il fait obligatoirement l'objet d'un débrayage énonciatif entre le plan 1 où est prédiquée la perception et le plan 2 où elle est développée. Le PDV représenté peut être un bon moyen d'objectiver le PDV du personnage, notamment s'il est introduit par le verbe « regarder " par lequel le narrateur présuppose, lui aussi, l'existence de l'objet perçu. Le PDV du narrateur serait par contre moins impliqué s'il avait choisi par exemple « voir » ou « avoir l'impression que » comme verbe de perception. Ce PDV représenté, introduit par le verbe "regarder », favorise donc l'immersion du narrateur, et par la même occasion du récepteur, dans les perceptions, marquées d'affects, du personnage ; il favorise donc l'empathie. Toutefois, la présence du tiret suivi du « et » marque une séparation entre la prédication du PDV et le PDV en luimême. Cette démarcation symbolise le fait que le paysage s'anime au moment où Élisa pose son regard sur lui; c'est donc bien l'affect du personnage qui influence la description.

À travers ces analyses, nous voyons que le choix des mécanismes de prise en charge est bien motivé par les effets pragmatiques (idéologique et pathémique) que le narrateur entend produire sur le récepteur. Toutefois, quel que soit le type de mécanisme choisi, il est toujours possible d'y inclure des variations dans l'articulation des PDV et la répartition des rôles joués par ceux-ci.

51 Par ailleurs, les effets pragmatiques généraux du récit dégagés à partir des données textuelles (subjectivèmes et confrontation des PDV) peuvent être précisés en étant mis en rapport avec les données extra-linguistiques relatives à la situation de communication du discours.

\section{Point de vue et effets pragmatiques en rapport avec la situation de communication du discours}

Comme dit plus haut, le sens à donner au récit découle des relations d'accord et de confrontation entre les idéologies des différentes instances. L'idéologie ainsi dégagée pourrait alors être mise en rapport avec le projet littéraire à l'origine du roman. Mais tous les romans ne véhiculent pas forcément une idéologie unifiée. Par ailleurs, rechercher un «message» potentiellement délivré par l'ouvrage est une démarche interprétative qui risque d'être réductrice par rapport à l'ensemble des trajets de signification qu'il est souvent possible de dégager. C'est pourquoi il convient de considérer qu'en amont, le projet littéraire (qui peut être caractérisé aussi bien politiquement et socialement que stylistiquement) peut également déterminer le choix de chaque subjectivème particulier et de chaque confrontation de PDV contenus dans le récit. Nous avons effectué en partie ce travail de mise en évidence de l'argumentation indirecte qui préside aux choix et à l'articulation des différents subjectivèmes et PDV, en identifiant les effets pragmatiques généraux potentiellement visés par ceux-ci. Il convient à présent d'utiliser ces données pour les confronter aux éventuels déterminismes de la situation de communication. 
53 Paru en 1937, chez Gallimard, avec l'aide d'Emmanuel Mounier et de Jean Paulhan, La Femme de Gilles se trouve au centre d'un conflit d'interprétation et d'une vive interrogation sur l'identité de Bourdouxhe que suscite notamment la difficulté à construire l'image de l'auteur. Ce conflit d'interprétation porte notamment sur la question de l'appartenance ou de la non-appartenance du roman aux mouvances populistes et féministes. Sur ce sujet, on peut citer les récents travaux de Jaques Dubois, Jaqueline Paque et Paul Aron réunis dans le collectif Relire Madeleine Bourdouxhe (cf. Kovacshazy, Solte-Gresser 2011).

Les résultats de notre analyse dialogique du roman nous poussent à rejeter la possibilité de telles appartenances dans l'idée que le roman illustre justement, avec compréhension, le choix posé par Élisa de vivre sa vie dans une totale abnégation. Le narrateur nous montre que l'absence d'intérêt de l'héroïne pour les aspirations politiques, sociales, individuelles, des hommes et des femmes (jusqu'au siennes propres), ne tient pas de l'ignorance ou d'une incapacité à comprendre ce monde, mais d'un refus, un rejet volontaire qui permet par ailleurs au personnage de développer une acuité perceptuelle beaucoup plus développée pour ce qui lui parait, à lui (et semblerait-il au narrateur), plus essentiel : la contemplation (et la sensation) du monde en lui-même, des paysages, du foyer, des personnes physiques, perception somme toute plus spirituelle qu'intellectuelle qui lui procure une sorte de plénitude. Prenons deux extraits pour appuyer notre propos :

(8) Devant un être semblable, Élisa se sent étrangement démunie : par où peut-elle toucher Victorine?

La jeune fille ne manque pas d'intelligence: Élisa se souvient qu'en classe elle était parmi les premières (que pourrait-elle trouver comme autre critérium...) Victorine a le sens de la justice: il y a des gens pauvres, il y a des gens riches, c'est injuste. Sens qu'Élisa ne possède pas d'ailleurs, quand Gilles lui fait part des revendications des camarades du syndicat, elle demande :

- Pourquoi demandez-vous cela?

- Parce que c'est juste, répond Gilles.

Alors elle renverse un peu la tête en arrière, elle rit.

- Qu'est-ce que ça veut dire « juste»! dit-elle.

Mais dans le cas qui pour l'instant la préoccupe, elle sent bien qu'il n'est pas question de justice ou injustice... que Victorine commette le mal, elle veut bien le comprendre, mais ce qui lui apparaît monstrueux, inexplicable, c'est cette allure angélique que la jeune fille conserve. (pp. 75-76)

(9) Elle les rejoignit bientôt et ils descendirent tous trois la route glissante et boueuse. Ils ne parlèrent pas. L'air était glacé. (...) Ils marchaient vite. Malgré la lourdeur de son ventre, Élisa n'éprouvait aucune difficulté à poser les pieds sur les pierres du chemin. Elle promenait son regard, vivement, sur la rangée de maisons, à droite puis à gauche, et ce regard rapide enregistrait toute chose avec acuité. Elle apercevait chaque petit glaçon sale qui scintillait dans la rigole, contre le trottoir ; elle voyait exactement où finissait le halo des réverbères. En passant devant une fenêtre éclairée, elle vit une femme penchée sur une table à demi desservie : elle eut le temps d'apercevoir son visage, ses cheveux, sa bouche, ses gestes, sa vie. Par ce regard qui avait duré tout juste les quelques secondes nécessaires à trois corps en marche pour briser un rectangle de lumière, Élisa connaissait cette femme.

Elle se dit que ces deux êtres qui marchaient à côté d'elle, à la même cadence et sur la même route, bien qu'ils vissent eux aussi les glaçons, le brouillard lumineux des lampes, les façades fermées ou les fenêtres éclairées qui nimbaient d'une triste lumière la vie des femmes, ils n'avaient de ces choses aucune connaissance. Et elle sentit monter en elle et réconforter son cœur une fierté profonde mais sans mépris. (pp. 23-24) 
Paul Aron (2011) interprète (8) comme une preuve de l'ignorance d'Élisa, de sa «myopie au monde », qui l'empêche de se réaliser en tant que personne (en référence à l'idéologie du personnalisme théorisée par Emmanuel Mounier), statut qu'elle parviendrait néanmoins à obtenir en se suicidant. C'est la raison pour laquelle le roman pourrait s'inscrire dans la mouvance féministe. Pourtant, une analyse polyphonique de l'extrait peut dévoiler qu'Élisa, tout autant que le narrateur, prend une posture ironique par rapport à la définition de la justice de Victorine et au fait que Gilles brandisse ce concept comme justification des revendications grévistes. Le passage correspond à un compte rendu des pensées d'Élisa où est employé le mécanisme du psycho-récit (souligné) qui explique les pensées d'Élisa exprimées en DIL (en italiques). On observe une nette incursion du narrateur avec la phrase entre parenthèses qui relève de son seul PDV puisqu'il remet en question le bienfondé du jugement d'Élisa. On est dès lors tenté d'interpréter l'ironie contenue dans la phrase suivante (c'est-à-dire dans la définition simpliste que Victorine donne de l'injustice) comme relevant du PDV du narrateur. Pourtant, nous soutenons qu'il persiste une ambigüité et qu'Élisa pourrait très bien, dans ce passage, avoir une posture ironique par rapport aux personnes, jugées intelligentes, qui développent un "sens de la justice " qu'elle-même ne comprend pas, non par ignorance, mais parce qu'elle juge ce concept vide de sens. L'ironie de cette phrase peut d'ailleurs être mise en parallèle avec l'attitude moqueuse d'élisa suite à la réponse de son mari qui brandit le mot «juste » pour toute justification. La réplique d'Élisa «Qu'est-ce que ça veut dire "juste"!», qui n'est d'ailleurs pas une véritable question, est loin d'être un aveu d'ignorance ; il s'agit tout simplement d'un désaccord quant à la validité de la notion-même de justice. Ce désaccord prend d'ailleurs tout son sens si on replace cette idée dans l'idéologie générale du personnage, empreinte de christianisme, et dans laquelle chaque homme doit accepter son sort et vivre dans l'abnégation (en témoignent plusieurs parallélismes effectués entre Élisa et les saints martyrs qu'elle contemple).

Ce sens de la justice que ne possède pas Élisa, et qu'elle semble plutôt ne pas vouloir posséder, est compensé par une acuité perceptuelle hors du commun lorsqu'il s'agit d'observer les personnes et le monde qui l'entoure, comme le montre (9). Le passage est composé de plusieurs PDV représentés auxquels le PDV du narrateur est étroitement lié. En effet, comme ce dernier choisit le verbe de perception, il se porte également garant des jugements contenus dans les modalisateurs soulignés. De ce fait, il confirme qu'Élisa possède bien un don. Élisa elle-même est consciente de ce don dont elle est fière et qui lui permet, encore une fois, de se différencier de Gilles et Victorine. Comme le montre le passage souligné en grasses, Élisa confère à ces perceptions le statut de " connaissances ». Nous sommes donc bien loin de l'image d'un personnage totalement aliéné, ignorant du monde et prisonnier de son destin.

Les extraits analysés précédemment sont également révélateurs de cette non-équivalence entre abnégation et aliénation, souvent confondues par les critiques.

Si Élisa choisit de renoncer à l'expression de ses rancœurs, c'est que dans l'ordre de ses priorités, l'amour de son mari passe avant tout (même avant ses enfants). Elle décide de le reconquérir par la voie de la manipulation. C'est ce qu'on observe en (2), (6) et (7).

59 En (2) les pantoufles chaudes symbolisent l'attention qu'Élisa porte à Gilles, dans une totale abnégation d'elle-même et de sa propre rancœur puisqu'elle sait que Gilles lui ment. Quant à la notification que choisit d'émettre le narrateur : «Gilles s'étire, étend les jambes, remue un peu les pieds dans les pantoufles chaudes » avant d'aboutir sur le DD 
« Il fait bon ici... dit-il, j'ai faim... », elle a pour but d'amener à juger la goujaterie de Gilles et la façon dont il profite impunément du bien que lui procure Élisa, tout en lui mentant. Là encore, la réponse "C'est prêt... » montre, en parallèle avec les pantoufles, que subvenir aux moindres besoins de Gilles prend, dans l'esprit d'Élisa, une place plus importante que ses plus graves préoccupations à elle. Cette scène peut, elle aussi, être interprétée comme symbolisant l'aliénation d'Élisa. Pourtant, on observe qu'Élisa détient une supériorité de savoir sur Gilles, supériorité qu'elle entend bien conserver et dont elle se sert pour exprimer de l'ironie avec le «Évidemment... ». En rapportant cette partie du $\mathrm{DD}$, le narrateur entend conférer un pouvoir à son personnage : si dans la diégèse Élisa est la seule à pouvoir cerner sa propre ironie, hors diégèse une complicité moqueuse est alors créée entre le personnage et ceux qui l'observent virtuellement, le narrateur et le lecteur. Il arrive pourtant que le narrateur montre l'infériorité du savoir d'élisa, comme en (3). Mais dans ce cas, il prend la précaution de montrer préalablement à quel point Élisa aime Gilles, créant une empathie pour l'hérö̈ne, de sorte que, comme nous l'avons déjà démontré, l'effet idéologique dégagé va à l'encontre de Gilles, et qu'aucune moquerie visà-vis d'Élisa n'est possible. Par ailleurs, (6) nous montre que si Élisa ne révèle pas ses doutes quant au fait que Gilles la trompe, ce n'est ni par peur, ni par ignorance, mais par choix, et ce choix fait partie d'une stratégie : elle veut mener Gilles à l'aimer de nouveau, à son insu, au moyen de "petits actes subtils ». Elle se pose donc en maîtresse de son destin et en manipulatrice de Gilles. En (7), enfin, Élisa désire succomber, l'espace d'un instant, à une forme de naïveté délibérée, s'imaginer que Gilles ne la trompe pas et retourner auprès de ses enfants qu'elle culpabilise d'avoir laissées seules. Avant de prendre sa décision, elle regarde alors le paysage. Les « formes noyées de nuit » et les «lueurs voilées de brouillard » symbolisent vraisemblablement le non-savoir dans lequel Élisa risque de s'abandonner. Or, de ces formes et lueurs «mont[e]nt à nouveau une tristesse infinie ». Tout apparait comme si le paysage portait conseil à Élisa qui, alors, se ravise et choisit de rester. ${ }^{12}$ Élisa choisit donc d'affronter la vérité, même si elle doit pour cela laisser ses enfants seules. Le narrateur s'emploie alors à expliquer ce choix, à prouver, l'amour qu'Élisa porte néanmoins à ses enfants. La responsabilité du narrateur dans la formulation et la disposition de ces psycho-récits justificatifs nous montre que ce dernier entend désarmer, par la force de la compréhension, les effets idéologiques négatifs susceptibles d'être produits par le fait qu'Élisa fasse passer son mari avant ses enfants. La dimension corporelle est de nouveau convoquée pour expliquer en quoi cet amour dépend de l'amour de Gilles et élisa.

Cette attention toute particulière accordée aux sens et à la dimension corporelle s'explique par l'acuité exceptionnelle avec laquelle élisa perçoit le monde qui l'entoure. Or, cette perception du corps est à de nombreuses reprises mise en rapport avec la valeur d'abnégation; c'est elle qui justifie les sacrifices auxquels se livre Élisa, en toute bonne fois. Ainsi en (3), Élisa se compare à Victorine en fonction de leur corps. Elle ne ressent alors aucune jalousie mais fait preuve de l'abnégation la plus totale puisqu'elle déduit logiquement qu'en vertu de son corps Victorine est plus apte à accompagner Gilles qu'elle-même. En (4), le narrateur et Élisa semblent partager le même désintérêt pour les propos que peut tenir Victorine, puisque ni l'un ni l'autre n'y réagissent. Par contre, une attention particulière est portée à la posture et à l'accoutrement de la jeune femme. Les deux faisant l'objet d'un jugement négatif de la part d'Élisa et du narrateur, parce que la nonchalance et la fausse élégance représentent en quelque sorte l'opposé de l'abnégation et bafouent la beauté naturelle (cette « fraîcheur » qu'a perdu Victorine). En (1), l'enfant que porte l'héroïne est désigné par la métonymie « poids », remplaçant de nouveau l'être 
par la dimension sensorielle et corporelle. Le fait que, tout au long du roman, Élisa ressente souvent la lourdeur pourrait être interprétée comme un signe de l'aliénation du personnage. Mais on observera que cette sensation de lourdeur s'accompagne tantôt d'émotions négatives, tantôt d'émotions positives. En l'occurrence, cette figure s'inscrit dans un cotexte immédiat positif : ce poids est "nouveau ", " porté fièrement " parce qu'il est le symbole de son union avec le " corps de Gilles». On remarque encore une fois la métonymie " corps de Gilles " pour "Gilles ». La question de l'abnégation prend tout son sens si l'on considère, que, pour Élisa, sa vie n'a de sens qu'à partir du moment où elle «fait corps » avec son foyer, son mari, ses enfants, ainsi qu'avec le monde, le paysage qui l'entoure. L'abnégation nécessaire à cette fusion avec le monde, exclut toute forme de velléité humaine. La perte de l'amour de Gilles et de son amour pour Gilles symbolise une rupture dans le rapport de continuité d'Élisa au monde: elle ne peut plus s'occuper du foyer, de ses enfants et elle ne peut tout simplement plus vivre. Sans cet amour et cette continuité, il n'est plus question d'abnégation volontaire mais, justement, d'aliénation. En effet, la sensation de lourdeur, omniprésente à la fin du roman, fait alors écho à sa faiblesse et à sa fatigue. Elle doit désormais porter, contre sa volonté, ce monde avec lequel elle ne fait plus corps. Ainsi, aux pages 151-152, même ses cheveux et son chignon deviennent trop lourds, symbolisant le fait qu'Élisa devienne étrangère à son propre corps et qu'elle n'ait plus la volonté et donc la force de le porter. Le narrateur lui-même cesse de faire corps avec son héroïne, au moment où celle-ci perd toutes ses perceptions. En témoignent les passages suivants :

(10) Debout près du haut panier d'osier elle est immobile, hagarde. Ses cheveux mal retenus se desserrent sur sa nuque. Oh! ne perds pas courage, Elisa! (...) la vie est partout... Attends... ne t'abandonne pas... Attends! la vie est sur le point de renaitre...

Mais elle ne pense pas, n'entend ni ne voit. (...)

Par la fenêtre basse du grenier on voit, au loin, les hauts fourneaux qui brûlent de toutes leurs flammes, de toutes leurs fumées. Elisa ne regarde pas au-dehors. (pp. 153-154)

61 Alors que dans l'ensemble du récit, le narrateur avait pour habitude de ménager des ambigüités quant à l'identification de la source des PDV. Ici, en notifiant que le personnage ne pense plus et ne perçoit plus rien, il nous empêche d'interpréter les deux passages soulignés respectivement comme un DDL où le personnage s'adresserait à luimême pour s'encourager, et comme un PDV représenté où les émotions du personnage animeraient le monde perçu. Qui plus est, le « on » qui précède la description du paysage rassemble cette fois le narrateur et le récepteur, mais exclut Élisa. Narrateur et récepteur sont ainsi détachés du personnage. Ce processus énonciatif symbolise également la rupture dans ce qui unissait Elisa au monde, la rendant étrangère et aliénée. En ce sens, le suicide d'élisa symbolise bien son refus de vivre dans l'aliénation, mais elle ne se considère, elle-même, comme vide et étrangère au monde qu'à partir du moment où son amour pour Gilles est rompu.

\section{Conclusion}

L'étude pragma-énonciative du récit, rendue possible grâce au repérage des subjectivèmes, à l'identification de leurs effets pragmatiques (idéologiques et pathémiques), à leur assemblage en PDV, ainsi qu'à l'identification des effets pragmatiques générés par les différents modes d'articulation entre PDV du narrateur et 
du personnage, nous a permis de remettre en question certaines interprétations du récit La Femme de Gilles. Elle permet en effet de dépasser les analyses narratologiques (focalisations) et sémiques traditionnelles, en identifiant plus précisément la portée pathémique et idéologique des relations énonciatives établies entre narrateur, personnage et lecteur.

\section{BIBLIOGRAPHIE}

Bibliographie

Barthes, R. 1982 [1968]), « L'effet de réel », Communication, n 11, p. 84-89.

Barthes, R. (1977), Fragments d'un discours amoureux, Paris, Seuil.

Bonhomme, M. (2005), Pragmatique des figures du discours, Paris, Honoré Champion.

Bourdouxhe, M. (2004 [1937]), La Femme de Gilles, Arles, Actes Sud.

Coste, D. (1980), « Trois conceptions du lecteur et leur contribution à une théorie du texte littéraire », Poétique. Revue de théorie et d'analyse littéraires, nº 41, p. 354-371.

Dobzhansky, T. (1966), L’homme en évolution, Paris, Flammarion.

Duchet, C. (1973), « Une écriture de la socialité », Poétique, n 16, pp. 446-454.

Ducrot, O. (1984), Le dire et le dit, Paris, Minuit.

Dumortier, J.-L. (2001), Lire le récit de fiction. Pour étayer un apprentissage : théorique et pratique, Bruxelles, De Boeck-Duculot.

Eco, U. (1985), Lector in fabula ou la Coopération interprétative dans les textes narratifs, Paris, Grasset. Hamon, P. (1984), Texte et idéologie. Valeurs, hiérarchie et évaluations dans l'œuvre littéraire, Paris, PUF.

Iser, W. (1985), L'acte de lecture. Théorie de l'effet esthétique, Bruxelles, Pierre Mardaga. Jouve, V. (1992), L'effet-personnage dans le roman, Paris, PUF.

Kerbrat-Orecchioni, C. (1980), L'énonciation, de la subjectivité dans le langage, Paris, Armand Colin. Kerbrat-Orecchioni, C. (1986), L'implicite, Paris, Armand Colin.

Klinkenberg, J.-M. (1990), Le sens rhétorique. Essais de sémantique littéraire, Bruxelles, Gref.

Klinkenberg, J.-M. (1996), Précis de sémiotique générale, Bruxelles, De Boeck.

Kovacshazy, C., Solte-Gresser, C. (dir.) (2011), Relire Madeleine Bourdouxhe. Regards croisés sur son œuvre littéraire, Bruxelles, Peter Lang.

Maingueneau, D. (2000), « Instances frontières et angélisme narratif. », Langue française, $\mathrm{n}^{\circ} 128$, p. 74-95.

Ouellet, P. et alii (1994), « La représentation des actes de perception : le cas de paraître », Les Cahiers de praxématique, $\mathrm{n}^{\circ} 22$, Université de Montpellier. 
Patron, S. (2009), Le narrateur. Introduction à la théorie narrative, Paris, Armand Colin.

Pottier, B. (2001), Représentations mentales et catégorisations linguistiques, Louvain-Paris, Éd. Peeters.

Rabatel, A. (2000), «Valeurs représentative et énonciative du "présentatif" c'est et marquage du point de vue ", Langue française, $n^{\circ} 128$, p. 52-73.

Rabatel, A. (2001), « La valeur de "on" pronom indéfini/pronom personnel dans les perceptions représentées ", L'Information grammaticale, $\mathrm{n}^{\circ} 88$.

Rabatel, A. (2003), « Le problème du point de vue dans le texte de théâtre », Pratiques, nº 119-120, p. 7-33.

Rabatel, A. (2004), Argumenter en racontant, Bruxelles, De Boeck.

Rabatel, A. (2008), Homo narrans. Pour une analyse énonciative et interactionnelle du récit, Limoges, Lambert-Lucas.

Rabatel, A. (2010), « Pour une narratologie énonciative ou pour une analyse énonciative des phénomènes narratifs ?», in Narratologies contemporaine. Approches nouvelles pour la théorie et l'analyse du récit, J. Pier et F. Berthelot (éds.), Paris, éd. des archives contemporaines.

\section{NOTES}

1. Dans cette étude, notre conception du narrateur sera celle des théories dites communicationnelles (cf. Patron 2009). Notre modèle est en effet basé sur des récits à la troisième personne, à narrateur extra-diégétique, ce dernier prenant en charge les paroles, pensées et perceptions des personnages dont il rapporte les points de vue (désormais PDV) et par rapport auxquels il peut sur- ou sous-énoncer sa propre subjectivité (cf. Rabatel 2008). D’après nous, il est donc possible de dégager les marques de subjectivité attribuables au PDV du narrateur et d'ainsi reconstituer un éthos discursif plus ou moins unifié et visible selon l'intensité de ce que nous appellerons l'«effet-narrateur ", c'est-à-dire la possibilité d'appréhender cette instance comme un «je» implicite doté d'une personnalité unifiée et identifiable.

2. Comme pour le narrateur, nous postulons qu'il est possible de dégager les marques de subjectivité attribuables au PDV du personnage et d'ainsi reconstituer son éthos, lui aussi plus ou moins unifié en fonction de l'intensité de l'« effet-personnage» (soit la cohérence de la personnalité représentée). Bien entendu il existe de multiples cas d'ambigüités quant à la source énonciative des subjectivèmes, ces ambiguïtés générant des effets pragmatiques particuliers (cf. infra).

3. Les théories internes de la lecture, que l'on nomme aussi théories de l'effet, ont élaboré deux concepts pour définir le lecteur présupposé par le texte : le lecteur modèle et le lecteur virtuel. Nous rejetons le concept de lecteur modèle d'Umberto Eco (qui correspondrait à l'instance disposant de compétences idéales la rendant capable de restituer l'ensemble des interprétations prévues par l'auteur) car nous considérons à l'instar de Didier Coste (1980) que le lecteur modèle est une construction du critique socio-historiquement déterminée. Ajoutons que cette perspective implique un retour à la conception d'une intentionnalité auctoriale érigée comme seule détentrice de l'interprétation dite valide. Or, tout comme les théories externes de la réception, nous estimons que le lecteur réel peut toujours enrichir le texte de sens nouveaux déterminés par le contexte socio-culturel dans lequel il s'inscrit. Selon nous, la notion de lecteur virtuel permet une conception plus souple de l'inscription du « rôle de lecteur » dans la structure du texte (cf. Iser 1985 : 72). En effet, il ne s'agit plus de reconstituer des contenus interprétatifs 
mais plutôt un rapport au lecteur tel que l'auteur se le représente, rapport identifiable en termes d'effets pragmatiques généraux. Ainsi, même s'il est impossible de prévoir l'impact précis du texte sur le lecteur réel, nous estimons qu'il est en revanche possible d'identifier le potentiel pragmatique des mécanismes énonciatifs en termes d'effets idéologiques et affectifs généraux.

4. Sauf mention contraire, c'est désormais à l'édition de 2004 de La Femme de Gilles de Madeleine Bourdouxhe que nous nous réfèrerons en ne mentionnant que les numéros de page. Il s'agit de l'histoire d'une femme au foyer dans le milieu ouvrier belge du XXe siècle, Élisa, dont le mari, Gilles, entretient une relation adultère avec la sœur d'élisa, Victorine. La construction narrative de ce récit est particulière; elle présente une forte intrication des PDV du personnage et du narrateur. En effet, alors que le PDV de l'héroïne y est pratiquement omniprésent, saturé en perceptions et en émotions, le PDV du narrateur s'exprime en sur- et en sous énonciation, manifestant, explicitement ou implicitement, une forte subjectivité idéologique et affective, et ce, jusque dans la représentation des pensées et perceptions censées appartenir intimement au personnage. Il nous semble que ces phénomènes d'intrication des PDV sont intimement liés aux nombreux conflits d'interprétation et à la vive interrogation sur l'identité de l'auteur suscités par ce roman, dès sa parution. Une analyse pragma-énonciative centrée sur l'étude des confrontations et articulations entre PDV du narrateur et du personnage nous semble, de ce fait, être la voie privilégiée pour proposer une nouvelle interprétation de ce récit.

5. Nous traitons des effets pragmatiques des subjectivèmes avant d'expliquer comment ceux-ci s'assemblent en PDV et avant d'aborder la problématique de l'hétérogénéité énonciative, parce que c'est notamment en fonction des concordances et discordances d'effets, liées à l'emploi de tel ou tel subjectivème, qu'il nous sera possible de rassembler ces derniers en PDV cohérents, attribuables à telle ou telle source énonciative.

6. Alors que pour Jouve le terme idéologie est synonyme de système de valeurs, Dumortier donne à ce concept une définition plus élargie ; il s'agit pour lui d'un «système de connaissances, de croyances, de valeurs et de normes de comportement » (2001: 137). L'effet idéologique du récit n'est dès lors plus seulement contenu dans les évaluations mais aussi dans les observations (énoncés de faits) et les prescriptions (énoncés d'opinions sur ce qui doit être fait ou sur les comportements à adopter). En effet, à travers ses observations, le narrateur cherche à persuader le lecteur de la réalité des faits observés et de la recevabilité de ses énoncés. Le lecteur remet alors en question ses propres croyances et connaissances. Les prescriptions sur le comportement à adopter dans telle ou telle situation imposent quant à elles des normes de comportement et des valeurs qui fondent ces normes. Elles contiennent donc des évaluations qui sont souvent exprimées de manière implicite (Dumortier 2001 : 141-142).

7. On peut toutefois se demander dans quelle mesure la conceptualisation discursive détermine elle aussi nos représentations du monde.

8. En effet, les focalisations interne, externe et zéro permettent seulement d'identifier le mode de régulation de l'information transmise. En concevant un modèle basé uniquement sur la catégorie du mode, en évitant d'intégrer à celui-ci toute considération relevant de la catégorie de la voix, et en niant du même coup l'importance du concept de focalisateur (c'est-à-dire de l'origine énonciative), Genette supprime également toute possibilité d'envisager (1) ce qui, dans la perception et la référenciation du monde fictif (subjectivèmes), renvoie à cette origine énonciative, à son éthos, à son PDV et dans le cadre qui nous occupe à sa relation avec les autres instances, et (2) les différents phénomènes de superposition et de juxtaposition des PDV du narrateur et du personnage ainsi que les effets pragmatiques générés par ces articulations.

9. Dans tous les extraits, les soulignements, les italiques et les grasses sont des ajouts de notre part.

10. Dans son modèle, Genette ménageait déjà une exception pour le phénomène qui consiste à raconter les comme un évènement et qu'il nommait «discours intérieur narrativisé » (2007: 174). 
11. Dans le cas de la prise en charge d'une pensée cognitive au moyen du DI, les verbes de cognition qui introduisent cette prise en charge impliquent toujours, comme le signale Rabatel (2008 : 506-508), un jugement du narrateur sur la pensée rapportée. Le jugement porte alors sur sa justesse (il savait que / il croyait que), sur son intégralité (il comprit que / il entrevit que) ou sur son objectivité (il admettait que / il s'imaginait que). Par ailleurs, Rabatel précise que le narrateur peut plus facilement effacer sa subjectivité lorsqu'il prend en charge des perceptions parce que ces verbes introducteurs-là (tels que voir, sentir, etc.) n'impliquent pas de jugement sauf en cas de négation ( il ne voyait pas que ») ou de modalisation (« il croyait voir que »).

12. Il est possible de voir dans cette mouvance du paysage, une allégorie du PDV bienveillant du narrateur, comme dans l'extrait suivant:

«Mais une banquette de bois restait dans chaque gloriette. Élisa pénétra dans la première, brusquement décidée à attendre quelques instants.

Elle s'assit sur le bois humide. Elle voulut passer les manches de son manteau mais elle craignait le froid qui, un instant, allait saisir ses bras nus et elle resta ainsi, son vêtement serré autour d'elle comme une couverture, petite masse sombre à peine perceptible dans l'ombre du berceau. La lueur d'un mauvais réverbère glissait entre les sarments défeuillés qui projetaient une ombre très douce sur la joue d'Élisa. "(p. 35)

Le passage souligné révèle une individuation du PDV du narrateur qui s'incarne en tant que sujet percevant (matérialisé par l'adjectif " perceptible »). Les termes « couverture », " petite masse », «berceau », « douce », « joue » entrent en résonnance pour comparer métaphoriquement Élisa à un enfant. La paronomase entre «sombre » et « ombre » mime formellement la fusion d'Élisa et du paysage. Dans ce cadre, et contenu de la métaphore Élisa-enfant assumée par le narrateur, la lueur du réverbère qui projette une ombre qualifiée de « douce » sur la joue d'Élisa, pourrait être assimilée métaphoriquement à un regard bien bienveillant du narrateur sur le personnage qu'il accompagne dans une épreuve difficile. Cette interprétation permettrait de justifier notre thèse de la corporalité, développée plus loin, selon laquelle narrateur, paysage et personnage font corps. Ce phénomène a pour effet de montrer l'empathie du narrateur et de provoquer ce même mouvement chez le lecteur.

\section{RÉSUMÉS}

Les instances internes au récit que sont le narrateur, le personnage et le lecteur virtuel entretiennent des relations, principalement idéologiques et affectives, que l'on peut décrire d'après une approche pragma-énonciative et rhétorique. Nous proposons une modélisation du système intra-relationnel $d u$ récit et une réflexion sur plusieurs problématiques que pose l'analyse de ces relations : le repérage des subjectivèmes relationnels, l'identification de leurs effets pragmatiques, leur assemblage en un point de vue émanant d'un sujet énonciateur particulier, les différentes articulations possibles entre les points de vue du narrateur et du personnage et leurs effets pragmatiques. D'après cette perspective nous proposons une nouvelle analyse et interprétation du récit La Femme de Gilles de Madeleine Bourdouxhe.

The internal instances in the narration such as the narrator, the character and the virtual reader maintain ideological and emotional relations which can be described according to a pragmaenunciative and rhetorical approach. We propose a model of the intra-relational system of the narration. The analysis of these relations raise the problem of the relational subjective 
investments: concerning most precisely their location, their pragmatic impact, their assembly in a point of view from an enunciative subject (whose éthos can be deduced) and the various possible linkages between narrator's and character's point of view with their pragmatic effects. According to this perspective, we propose a new analysis and interpretation of the narrative $L a$ Femme de Gilles of Madeleine Bourdouxhe.

INDEX

Index chronologique : XXe siècle

Mots-clés : narrateur, personnage, lecteur, énonciation, pragmatique, dialogisme, polyphonie, analyse du discours

Keywords : narrator, character, reader, enunciation, pragmatics, dialogism, polyphony, discourse analysis

Index géographique : Belgique

\section{AUTEUR}

\section{EMILIE GOIN}

Université de Liège, Département Sciences du langage et Rhétorique 